\title{
Les chroniques : étude exploratoire d'un genre d'écriture (très) populaire sur le net
}

\author{
Violaine Bigot ${ }^{1, \mathrm{a}}$, Nadja Maillard ${ }^{2}$ et Patricia Lambert ${ }^{3}$ \\ ${ }^{1}$ Université Paris 3, EA 2288 Diltec \\ ${ }^{2}$ Université d'Angers, Ceriec EA \\ ${ }^{3}$ ENS-IFE UMR 591-Icar
}

\begin{abstract}
Résumé. Depuis une dizaine d'année, des jeunes (surtout des femmes) racontent la fin de leur adolescence et leur entrée dans la vie adulte, sous la forme de récits à épisodes postés au fil des jours sur les réseaux sociaux. Un lectorat s'est constitué autour de ces écrits qui peuvent rassembler plusieurs (dizaines de) milliers d'abonné-e-s. Ces textes constituent donc un «espace littéraire », identifiable sur la toile et repérable grâce au nom de «chroniques». Cet article revient sur les questions méthodologiques que pose la constitution et l'étude d'un corpus à travers le prisme de la notion de "genre discursif». Pour étudier cette pratique discursive naissante, ancrée dans les réseaux sociaux, et délimitant une communauté d'autrices/auteurs et de lectrices/lecteurs, très active, nous avons inclus au corpus les métadiscours que suscite cette pratique. La constitution de ce corpus «bi-face» nous conduit à mobiliser et à interroger la distinction émique/étique en analyse du discours. C'est dans cette double perspective étique/émique que le corpus est ensuite exploré. L'approche par le "genre» nous permet d'envisager certains jeux de parenté, voire de filiation avec d'autres pratiques discursives dont on peut faire l'hypothèse qu'elles constituent une forme d'interdiscours des chroniques. En reprenant le corpus à travers différentes catégories d'analyse classiquement définies en analyse du discours (développement thématique, structure compositionnelle, ancrage énonciatif, procédés d'écriture), nous montrons quelques caractéristiques qui contribuent à la stabilisation du genre mais aussi interrogent certaines catégories d'analyse.
\end{abstract}

\begin{abstract}
Chroniques : exploratory research of a new speech genre developed on the internet. For the last ten years, young people (young women especially), living in multicultural suburbian areas, have been telling, on the internet, the life story of their teenage years and their transition into adult life. They share their story on social networds, publishing the episodes day after day. These stories, named "chroniques" (chronicles) by the writers and the readers, can reach an audience of several thousands of (mainly female) readers. This article is an attempt to show that Chroniques constitute a new speech genre, born on the internet, where it federates a wide speech community of writers and readers. The study is based on a corpora including not only the text of the story-telling itself but also discourses produced by the members of the speech community inside the community space (comments of episodes published
\end{abstract}

\footnotetext{
${ }^{a}$ Auteur de correspondance : violaine.bigot@univ-paris3.fr
} 
by the readers for example) or ,on its boarders (for example webpages created by "fans" who write literary critiques of chroniques, share links to chroniques, list them in repertoires...) Combining an etic and an emic approach, this article analyses recurrent caracteristics of the chroniques, focusing especially on "thematic content, style, and compositional structure" (Bakhtin) and on questions about the of authorship.It aims at defining the "genre contract" that federates the speech community of writers and readers of "chroniques".

\section{Introduction}

Depuis une dizaine d'année, des jeunes femmes (rarement des jeunes hommes) racontent la fin de leur adolescence et leur entrée dans la vie adulte, sous la forme de récits à épisodes postés au fil des jours sur le réseau social Facebook. Ces écrits numériques peuvent rassembler plusieurs milliers d'abonné-e-s, dont une large majorité de (jeunes) femmes. Ils abordent des thématiques relatives au genre autobiographique (amour, mort, amitié, liens familiaux,...), et d'autres, plus liées à leur contexte de production (vie dans la "cité », expérience de la migration, séjours au «bled», chômage, transactions illicites, religion, etc.). Ces récits constituent un « espace littéraire » d'autant plus facilement repérable dans la masse de littéracie sur le web qu'ils y apparaissent comme une catégorie spécifique : les « chroniques ».

Notre projet s'est développé dans le cadre d'une réflexion sociolinguistique sur ce que ces chroniques sont susceptibles de nous apprendre au sujet de la «compétence de communication » (Hymes, 1984) de leurs autrices. La question de l'émergence et de la stabilisation d'un genre (para)-littéraire s'est imposée à nous dès notre première exploration des textes. Aussi, en amont des questions sociolinguistiques que pose ce corpus sur les pratiques langagières de jeunes, la littéracie numérique, l'hétérogénéité et le changement linguistique, avons-nous voulu l'étudier à travers la notion de "genre discursif» pour montrer comment celle-ci a pu contribuer à la constitution du corpus et en quoi l'inscription de ces textes dans les réseaux sociaux conduit à interroger, d'un point de vue méthodologique, la question de l'étude des genres.

Comme le rappelle Moirand, tout travail sur des données discursives nous confronte à la notion de "genre », en nous invitant à nous interroger sur les critères qui permettent de « rassembler des textes ou des interactions, que l'on voudrait à la fois comparables et contrastables » (Moirand, $2003: 1-2$ ). Nous nous interrogeons donc sur la «comparabilité » de ces textes publiés sur internet sous le nom de "chronique » et recherchons, dans les textes de chroniques elles-mêmes, mais aussi dans les commentaires publiés par leurs lectrices, «ce qui fait genre» dans cette nouvelle forme d'écriture. Dans une première partie, nous revenons sur les questions de méthode que pose l'étude d'un genre émergent sur le net. Nous explicitons le mode de constitution du corpus et revenons sur la manière dont il interroge la distinction émique/étique (dans la perspective développée par Olivier de Sardan, 1998). Cette distinction nous a semblé particulièrement pertinente pour analyser, dans la suite de l'article, les caractéristiques des chroniques qui constituent les termes du contrat de genre liant plus ou moins implicitement les membres de la communauté discursive des autrices/auteurs et lectrices/lecteurs de chronique ${ }^{1}$. 


\title{
2 Enjeux méthodologiques d'une recherche sur un genre d'écriture (para)littéraire du web
}

\subsection{La constitution du corpus}

Le genre peut être défini comme un ensemble de représentations cognitives (au niveau individuel) et de représentations sociales (partagées collectivement) qui orientent les pratiques discursives des locuteurs tout autant qu'elles se construisent à partir de ces pratiques. Ces représentations, qui concernent notamment ce qui peut être dit, par qui, comment, dans quel contexte (moment, lieu/support) et dans quel but, prennent la forme de règles d'obligations, de possibles et d'interdictions concernant le dicible (Von Müchow, 2010) et déterminent des « horizons d'attente » (Jauss 1978) chez le « récepteur».

Identifier les normes communicatives qui régissent un genre de discours implique donc de circonscrire un corpus dont on veut montrer les règles communes, alors que l'identification de ces règles communes constitue l'objet même de l'étude des textes rassemblés. Cette interrogation sur la nature circulaire de tout travail sur les genres discursifs est au cœur de la réflexion que développe Von Münchow sur la notion de genre discursif dans les recherches d'analyse contrastives de discours. Rappelant que cette difficulté a entre autres été pointée par Kerbrat-Orecchioni en 1994 et par Beacco en 2004, elle propose de la dépasser en distinguant genre étique et genre émique, sur le modèle de l'opposition entre « texte étique et texte émique » (Harveg 1971 cité par Von Münchow 2010) :

\begin{abstract}
«La démarche est la suivante : en choisissant des documents relevant de ce qu'on pense être un même genre, l'on aborde le genre dans une perspective étique; l'analyse met alors à la disposition du chercheur les critères linguistiques et extra-linguistiques pour définir le genre émique, concept qui permet de rejuger l'attribution étique du caractère générique à tel ensemble de documents, voire de reconsidérer la pertinence de l'analyse elle-même.» (Von Münchow 2007 : 111)
\end{abstract}

Parmi les critères auxquels on peut avoir recours pour "délimiter le "genre étique" en amont de l'analyse », elle évoque les «désignations ordinaires », qui ont joué un rôle fondamental dans la constitution de notre corpus.

En effet, après avoir découvert ${ }^{2}$ l'existence des «chroniques » sur internet, nous avons voulu mesurer l'ampleur du phénomène et explorer ce corpus potentiel. Nous nous sommes tout d'abord appuyées sur la désignation ordinaire (ou vernaculaire) "chronique », démarche qui s'est révélée très efficace pour repérer des productions discursives a priori comparables sur le net, surtout lorsqu'on le croisait avec le support de diffusion (Facebook, et dans une moindre mesure Skyblog ). Mais en entrant ces mots clés dans les moteurs de recherche, nous avons vite découvert que les chroniques y faisaient l'objet d'un métadiscours abondant et il nous est apparu que ce métadiscours, produit par la communauté discursive des chroniqueuses et de leurs lectrices, sont également des données pertinentes pour appréhender les représentations sociales du genre. Ces métadiscours constituent aussi un matériau précieux pour répondre, dans la suite de nos recherches, à la question des compétences discursives des autrices et des lectrices manifestées dans les chroniques. Nous avons donc choisi de construire un corpus bi-face, constitué à la fois des chroniques elles-mêmes et des discours produits à propos des chroniques sur les réseaux sociaux du net. 


\subsection{Présentation du corpus}

Nous avons constitué un premier corpus exploratoire à partir d'une sélection de chroniques opérée par des lectrices dans une liste intitulée « top 10 » et placée sur une page Facebook («La folie des chroniques ») rassemblant une communauté de lectrices de chroniques. Malgré l'intitulé de la liste, ce sont seulement 7 chroniques qui y sont recensées, dont 6 ont été étudiées $^{3}: 1$. Chronique de Soraya, des ténébres à la lumiére ; 2. Chronique de Sabrina love d'un renoi ; 3. Chronique de Sabrina : Cerise sur le Ghetto; 4. Chronique : La Vida à La Cité ; 5. Chronique de ma vie du passé et en direct ; 6. Chronique d'un Boug In Love.

Pour ces six chroniques, nous avons analysé de manière systématique les parties 1 et 2 , qui en constituent l'incipit, ainsi que les parties 19 et 20 , qui prennent place à un moment où le récit est déjà lancé. Deux autres chroniques, choisies de manière aléatoire parmi des chroniques très lues sont venues compléter ce corpus exploratoire. Elles ont fait l'objet d'une lecture intégrale et d'une analyse plus large : 7. Petite Cendrillon amoureuse du prince $d u$ Ghetto et 8 . Zairoise love d'un rebeu" ${ }^{4}$.

Nous avons enfin intégré au corpus des discours «méta» tenus sur les chroniques par les chroniqueuses et leurs lectrices. Ont ainsi été retenus, de manière ponctuelle, des commentaires postés à la suite des épisodes des chroniques de notre corpus ainsi que les échanges au sein d'un fil de discussion du forum du site «ma-bimbo.com». Intitulé «(littérature Internet) Le monde des chroniques », ce forum compte près de 200 messages, postés entre le 28 juillet 2012 et le 27 avril 2015. Nos analyses prennent aussi appui sur 14 pages Facebook (dont la page «La folie des chroniques », citée ci-dessus) et 2 Skyblogs (créés entre mai 2011 et août 2013) administrés par des lectrices qui y recensent, classent, archivent et commentent des chroniques existantes. Ces « répertoires de chroniques » ou «chronicothèques» (nom que se donne l'un de ces répertoires) regroupent ainsi des communautés de passionnées qui peuvent y retrouver des chroniques ayant parfois disparu de la toile, échanger sur leurs lectures, lancer des avis de recherche pour une chronique ou un type particulier de chroniques. Ces répertoires de chroniques permettent de "ranger» les chroniques dans des albums, auxquels les administratrices des pages donnent un nom indiquant le critère commun aux chroniques qui y sont rassemblées. Les 14 répertoires de chroniques sur Facebook nous ont donné accès à près de 80 albums ${ }^{5}$, chaque album proposant des liens vers une «série " de chroniques (de quelques-unes à plus de cent pour les albums les plus riches).

Enfin, pour appréhender l'ampleur du phénomène et mieux cerner ce qui permet d'y voir l'émergence d'un genre discursif, nous avons également inclus d'autres discours " méta ", produits quant à eux par des observateurs attentifs aux pratiques des réseaux sociaux en marge de la communauté des autrices et lectrices. Nous avons pour l'heure retenu cinq articles $^{6}$ et une parodie de chronique

L'idée d'ouvrir notre corpus à ces discours qui thématisent notre objet de recherche s'est imposée pour les raisons évoquées plus haut. Des analyses discursives plus poussées nous conduiront sans doute à conclure à ce que Cislaru et Sitri (2009), reprenant Moirand, nomment l' «impossible clôture » du corpus. Ces analyses révèleront sans doute aussi l'intérêt d'adopter une démarche « cadrative » (Cislaru \& Sitri, 2009) du corpus pour notre projet d'étude de la compétence de communication des locutrices/locuteurs de la communauté discursive constituée autour des chroniques.

Ce choix d'associer au sein du corpus les textes des chroniques et les textes produits à propos des chroniques est enfin motivé, dans cette première étape, par la volonté de combiner une perspective étique et une perspective émique sur le genre. Ces adjectifs sont d'abord utilisés ici en référence à Olivier de Sardan (1998), dans un article où il reprend et 
prolonge les débats qui se sont développés, depuis la proposition de Pike, de transposer l'opposition étique/émique au champ de l'anthropologie.

\subsection{Perspective émique et perspective étique pour l'étude des genres discursifs sur internet}

Dans le champ de l'anthropologie, la distinction entre approche étique et approche émique peut opposer les savoirs produits par les chercheurs sur les comportements d'une communauté (étique) et les savoirs produits par les membres de la communauté en question (émique). Olivier de Sardan, reprenant un certain nombre de critiques qui ont pu être adressées à l'opposition étique/émique telle que Harris l'a développée à partir des travaux de Pike, rappelle qu'il n'y a pas de solution de continuité entre savoirs savants et savoirs ordinaires et que les savoirs savants sont donc une forme particulière de savoir ordinaire (Olivier de Sardan, 1998). Cette opposition peut également être située sur le plan des données produites. Sur le plan émique, les données sont produites par les membres de la communauté observée (dans les discours qu'ils adressent au chercheur pour partager avec lui leurs savoirs non-savants). Les données produites par le chercheur (observation et comptage de comportement) constituent alors le plan étique de recueil de données. L'opposition est parfois résumée par discours vs comportements. La validité des données émiques a pu être critiquée pour les écarts qu'elles présentent avec les données produites par le chercheur (écarts entre pratique et discours sur la pratique) mais aussi pour les problèmes méthodologiques qui se posent dès que les informateurs d'une enquête $\mathrm{s}$ 'adressent à un chercheur pour répondre à ses questions.

Lorsqu'on réintroduit l'opposition dans le champ des sciences du langage et qu'on la mobilise en analyse du discours pour penser une question comme celle des genres de discours, les « comportements » que l'on observe (dans notre cas, l'écriture des chroniques) sont de nature langagière. Ce sont donc des "discours» que l'on analyse dans une perspective étique et les données que l'on peut recueillir dans une perspective émique sont des «métadiscours ", qui prennent donc pour objet le discours lui-même ou la pratique discursive qui le fait émerger. Dans notre recherche, ces métadiscours peuvent intervenir dans le cours de l'activité langagière elle-même, lorsque les autrices catégorisent leurs textes comme des «chroniques », par exemple dans les titres. D'autres métadiscours peuvent nourrir cette perspective émique sur les chroniques, notamment lorsque ces dernières sont prises comme objet de discours à part entière (sur les forums ou dans les commentaires des épisodes évoqués ci-dessus).

Le caractère novateur d'un genre sur le net génère des échanges méta au sein de la communauté discursive (constituée dans notre cas des chroniqueuses et de leurs lectrices) : des métadiscours très riches se trouvent ainsi à la disposition du chercheur sans qu'il les ait sollicités. Ces discours méta sont d'autant plus denses et identifiables que le genre de discours se développe de manière privilégiée sur des réseaux sociaux. Les membres de la communauté échangent sur leur compréhension de la nouvelle pratique discursive, partagent leurs lectures de textes du genre, produisent des critiques. Internet donne un accès direct au chercheur sur ces échanges qui révèlent la perception que les membres de la communauté ont des caractéristiques du genre et de son éventuelle stabilisation. Il s'agit de discours « situés », qui doivent donc être " resitués », comme tout discours, au moment de leur analyse, mais leur prolixité et le fait qu'ils soient adressés à des membres de la communauté offre des données riches et «écologiques » pour appréhender l'émergence de ce genre d'un point de vue émique 


\subsection{Associer perspective émique et perspective étique pour identifier les caractéristiques du genre « chroniques »}

L'établissement des caractéristiques du genre que nous avons proposons s'appuie donc sur une étude systématique du corpus des huit chroniques présenté plus haut, corpus complété par des discours produits dans ou hors de la communauté des chroniqueuses et lectrices de chroniques, dont nous avons vu supra qu'ils constituaient des données émiques.

D'autres discours, qui ne font pas à proprement parler partie du corpus, seront convoqués dans le courant des analyses. Ces discours, proches de ceux des chroniques, du point de vue des thèmes traités et/ou de la forme compositionnelle et stylistique, sont mobilisés pour faire émerger, au-delà des points communs, des différences qui permettent de délimiter le genre chronique. Là encore, la sélection de ces discours "proches » se justifie par des données produites émiquement. Ainsi, le regroupement d'épisodes, par les chroniqueuses elle-même, en séries qu'elles dénomment "saison " " contribue, avec un faisceau d'autres traits, à inscrire le genre des chroniques dans une proximité avec les feuilletons et les séries télévisées dont il devient intéressant de voir comment elles s'en distinguent. Mais notre connaissance d'autres genres discursifs nous invite aussi à établir des points communs et faire émerger des différences avec des discours avec lesquels les chroniqueuses n'ont peutêtre jamais été en contact direct. Il s'agit alors d'une confrontation à valeur heuristique, qui relève d'une approche étique. Il ne s'agit pas alors de chercher à identifier des formes présentes dans la bibliothèque intérieure des chroniqueuses et qui pourraient nourrir leur écriture mais simplement de procéder, par comparaison, pour faire émerger les traits saillants des chroniques, en tant que genre à part entière. Ce travail de comparaison, inhérent à toute analyse qui vise à distinguer les caractéristiques d'un genre, est présent dans les différentes étapes de l'analyse qui se développe dans les parties 3 et 4 .

La première entrée que nous avons retenue pour identifier les caractéristiques du genre des chroniques est celle de la dénomination (partie 3). Nous l'avons abordée selon une perspective émique, en regardant comment la communauté discursive mobilise le terme de " chroniques », mais aussi dans une perspective étique, en recherchant, dans d'autres productions discursives antérieures au nom proche, des traces d'une possible filiation discursive.

Dans la partie 4, notre analyse s'appuie sur la confrontation des modèles d'analyse des genres discursifs proposée par Moirand (2003). Nous avons organisé notre étude autour des trois pôles dégagés par Bakhtine en dégageant des caractéristiques stylistiques, compositionnelles et thématiques qui nous semblaient saillantes dans le genre «chroniques », parce que récurrentes et partagées par l'ensemble des textes du corpus de chroniques. Nous nous sommes également intéressées aux caractéristiques de l'ancrage énonciatif des textes (statut des énonciateurs et co-énonciateurs et circonstances temporelles et locales de l'énonciation), catégorie d'analyse proposée notamment par Maingueneau (1996).

Sur le plan de la composition du texte, nous nous sommes intéressées au caractère très stéréotypé des incipit et à l'utilisation du format «feuilleton » pour la construction des récits. L'étude de la place et des formes des dialogues dans les chroniques, de la présence d'un pacte autobiographique et l'instabilité des textes de chroniques entre les plans du discours et de l'histoire constituent deux autres caractéristiques identifiées, qui relèvent principalement d'une analyse stylistique et d'une analyse sur le plan énonciatif. Enfin, nous nous sommes intéressées aux thématiques récurrentes développées dans ces récits. 


\section{Quand la dénomination fait genre}

Le terme de «chronique » est employé à la fois par les autrices, qui étiquettent ainsi leur récit de manière à le rendre visible sur leur page Facebook, et par les lectrices qui interviennent soit directement sur les pages Facebook où sont publiées les chroniques soit dans les autres espaces du web dédiés aux chroniques et présentés ci-dessus.

\subsection{La dénomination du genre : un critère à la fois central et discutable}

L'emploi du terme chronique relève de ce que Petit et Beacco nomment une « verbalisation métalinguistique non savante des représentations ordinaires des types de discours » (Petit et Beacco, 2004: 87) et témoigne "d'une activité analytique et classificatoire préalable à toute classification scientifique » (Petit et Beacco, $2004: 88$ ).

Ainsi, les titres des chroniques comportent systématiquement ce terme, inscrivant de facto le texte produit dans le genre ainsi affiché. Cette inscription explicite dans le genre se retrouve dans tous les titres des chroniques de notre corpus. 7 d'entre eux prennent la forme : chronique de (+ syntagme nominal) et, pour le dernier, l'appartenance générique est précisée entre parenthèses après le titre : Cendrillon, amoureuse d'un prince du ghetto (chronique). Par ailleurs, cette catégorisation explicite se retrouve également dans les « répertoires » qui leur sont consacrés. Par exemple, la chronicothèque qui a servi de base à la constitution de notre corpus s'intitule «La Folie des chroniques » et propose de ranger les chroniques dans différentes catégories, comme «chroniques qui parlent du Dine » ou «chroniques finies ».

Tous ces usages du terme « chronique » confirment que, pour la communauté discursive, il existe un genre clairement identifié.

La dénomination " chronique » fournit un affichage générique qui contribue à rendre le récit visible dans la profusion des écrits de la toile, lui permet d'y prendre place dans un espace (para) littéraire clairement identifié et donne un «statut » à son autrice qui devient de facto une " chroniqueuse ». La rapidité avec laquelle semble s'être imposée cette dénomination doit probablement aussi être reliée à la logique des moteurs de recherche qui pousse celui qui veut être retrouvé dans les fils de la toile à anticiper le repérage de son texte par mots clés, l'appartenance à un genre constituant, pour toute production artistique, une des premières formes d'étiquetage possibles. Bien sûr, le fait qu'un nom de genre empirique - en l'occurrence ici celui de «chronique »- se soit imposé au sein d'une communauté discursive ne suffit pas à garantir qu'il désigne bien un et un seul genre. Les noms génériques peuvent fonctionner comme des «leurres» (Mourlhon-Dhalies, 2007 : 20) et on a parfois tendance à qualifier de "nouvelle » toute forme de production textuelle encore non-étiquetée sur internet sans forcément voir qu'elle peut être inscrite dans des genres qui se sont développés bien avant, en dehors de la toile.

\subsection{Les discours sur la naissance, la gloire et la décadence du genre}

Les échanges qui interrogent la question de la naissance et de la mort du genre et son éventuel «âge d'or » constituent également des lieux de négociation autour de ce que peut recouvrir la dénomination et contribuent à la potentielle stabilisation du genre.

Dans les discours «méta» produits à propos des chroniques, chroniqueuses et lectrices identifient les chroniques comme un phénomène historique, né à un moment donné, qui évolue, et tend peut-être aujourd'hui à perdre de l'ampleur. C'est par exemple ce que fait Axellya, qui est à l'initiative du fil de discussion consacré aux chroniques dans le 
forum « ma-bimbo.com », lorsqu'elle essaie d'identifier le moment où les chroniques sont apparues sur la toile ${ }^{9}$ :

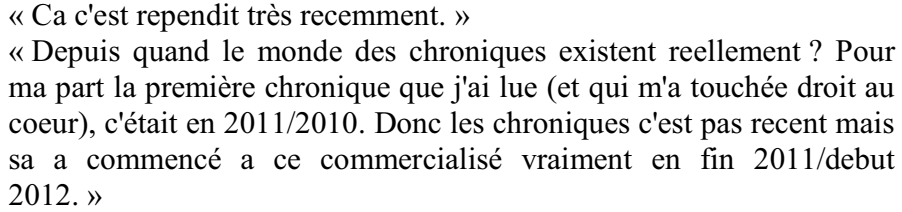

Cette mise en avant de la dimension diachronique du genre conduit aussi certaines lectrices - et chroniqueuses - à thématiser une forme de désaffection vis-à-vis des chroniques et / ou à regretter un "âge d'or» des chroniques. Comme tout discours nostalgique, il semble d'ailleurs se développer très précocement dans l'histoire des chroniques elles-mêmes, comme en témoignent par exemple les échanges entre la chroniqueuse de Zaïroise love d'un rebeu et ses lectrices. Elle poste ainsi le 24 juillet 2012 le message suivant :

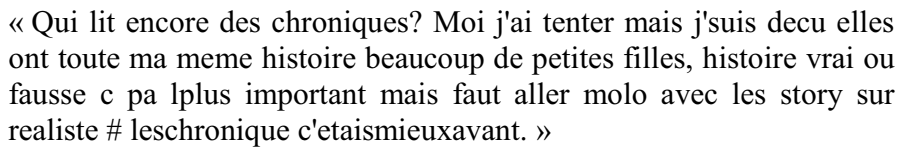

Et les commentaires suivants lui font écho : «Moi j'étais accro j'en lisais plein mais les chroniqueur(se) partent trop en couillent trop de FAKE ça donne même plus envie d'en lire ... », ou encore :

«Lol c'est exactement ce que je pensais "les chroniques c'etait mieux avant" jcomprend $\mathrm{mm}$ pas concept d'inventer une chronique ! Et certaines chroniques sont ecrient en mode sms du debut a la fin faut pas abuser la!»

On trouvera dans ces discours des résonnances avec une conception quasi « biologique » du genre, telle que Brunetière a pu par exemple la développer, sa théorie de l'évolution des genres pouvant être selon les mots de Schaeffer «considérée comme l'aboutissement extrême du paradigme biologiste dans le domaine de la classification littéraire » (1989 : 48).

La présence des ces discours sur l'origine et la décadence des chroniques nous indique que pour cette communauté discursive, nous ne sommes pas face à un genre que Maingueneau qualifierait de « routinier». En effet, pour les genres routiniers (qu'il oppose aux genres « auctoriaux »), Maingueneau considère que cela «n'a pas grand sens de se demander qui les a inventés, où et quand ; un érudit - à supposer que ce soit possible - peut toujours retrouver qui a publié le premier journal quotidien, qui a fait le premier "talk-show" à la télévision ou la première ordonnance médicale, mais ici la question de la source n'est pas pertinente pour les usagers » (Maingueneau, 2007: 7). La question de la source, ou du moins l'inscription dans une histoire de discours, est donc thématisée - et jugée comme pertinente - par la communauté discursive qui rassemble les chroniqueuses et leurs lectrices. Ceci constitue un indice parmi d'autres que cette communauté discursive inscrit les chroniques du côté du genre «auctorial» et possiblement dans le champ du littéraire (Maillard et Bigot 2015).

\subsection{Une histoire de filiation}

Poursuivant la perspective émique adoptée ci-avant pour voir comment la communauté discursive commente la naissance du phénomène, nous pouvons nous interroger sur les genres avec lesquels une filiation est établie. Si on entre dans les discours " sur» les chroniques, on découvre que l'intertextualité ne fait guère l'objet de discours. La question 
du plagiat interchronique est discutée, mais les liens avec d'autres genres de productions discursives, qui pourraient nourrir la production des chroniques, ne sont jamais commentés.

Dans un premier temps, nous avons donc complété les informations que nous donnaient les discours de la communauté discursive sur la naissance du genre, en enquêtant sur sa présence sur la toile, dans une perspective cette fois tout à fait « étique », pour essayer de voir si des co-occurrences passées des termes «chroniques » et "banlieues 》 ou " cité » pouvaient nous aider à comprendre la filiation de ce genre nouveau.

Précisons que l'usage du terme «chronique » qui est fait dans notre corpus n'est pas sans lien avec les définitions que l'on donne du terme pour son emploi dans la littérature du Moyen Âge : «œuvres historiques privilégiant l'ordre chronologique des faits dont on conserve la mémoire », ou dans le «sens dérivé » qu'on donne au terme à partir du $19^{\text {ème }}$ siècle : «cycle de romans retraçant l'histoire d'une famille» (Lucken, 2002: 90). Plus généralement «l'usage du terme se fonde sur "l'idée d'une suite de faits ": relatée au jour le jour, elle donne la chronique au sens journalistique, retraçant l'ordre historique, elle suscite la "chronique romanesque" mais surtout l'idée du témoignage et du commentaire y est devenue majeure » (Lucken, 2002 : 91). Le contact des membres de la communauté discursive avec cette production littéraire n'est cependant pas attesté, et l'on peut plutôt faire l'hypothèse que c'est par des relais que le terme "chronique »s'est imposé pour désigner ces écrits autobiographiques.

Notre recherche croisant les mots clé de « chronique » et de «banlieue » ou « cité » montre que le terme est fréquemment utilisé avant l'apparition des premières chroniques sur la toile pour des blogs / sites qui ont pour but de rendre compte, de témoigner au jour le jour du quotidien. Nos recherches attestent que dans la seconde partie des années 2000, la catégorisation «chroniques » a été à plusieurs reprises utilisée pour intituler des blogs ou des enquêtes journalistiques portant sur l'univers de la banlieue ${ }^{10}$. On notera aussi, dans le même ordre d'idée, l'édition en 2007 d'un ouvrage collectif, Chronique d'une société annoncée. Cet ouvrage regroupe un collectif d'auteurs qui «partagent le goût d'une littérature du réel, sociale et revendicative, [militent] pour une reconnaissance sensible des territoires en souffrance et de ses habitants, et plus largement pour tous ceux qui n'ont pas voix au chapitre de ce pays ${ }^{11}$ ». On se contentera de formuler avec prudence l'hypothèse d'une parenté discursive entre ces écrits de la fin des années 2000, explicitement reliés au genre littéraire de la «chronique » et produits dans le but de «témoigner » du quotidien d'un espace social mal connu du lectorat visé et les chroniques de notre corpus, qui construisent une communauté essentiellement constituée de lectrices pour qui l'univers socio-urbain des «cités» semble familier ${ }^{12}$. Le genre des chroniques est donc identifié comme tel via l'emploi, et la circulation, d'une dénomination spécifique, via aussi des discours tenus sur ce genre au sein d'une communauté discursive donnée. Mais l'analyse des productions discursives étiquetées comme "chronique » confirme-t-elle l'émergence et la stabilisation d'un genre discursif nouveau ? L'utilisation du terme " chronique » par les autrices de récits autobiographiques sur le web constitue une forme d'auto-catégorisation du texte (mon récit appartient à un genre) dont il convient d'examiner dans quelle mesure il inscrit le texte publié dans un genre stabilisé. Pour répondre à cette question, nous proposons donc d'analyser les textes du corpus pour en dégager des caractéristiques stylistiques et compositionnelles, thématiques et énonciatives. 


\title{
4 Construction compositionnelle, caractéristiques stylistiques et contenu sémantique
}

\subsection{Les incipits : un format très routinisé}

Les incipits répondent à des conventions d'écriture partagées par la communauté des chroniqueuses. On peut donner celui de Sabrina, Love d'un renoi comme exemple canonique.

\author{
« Coucou les frères et soeurs, j'ai décidé à mon tour de me lancer pour \\ écrire une chronique, donc une ptite présentation s'impose mes \\ chouchous \\ émoticône wink \\ Je m'appelle Sabrina j'ai 24 ans (presque 25 mais psychologiquement \\ le 24 passe mieux lol) (...) \\ Je vis donc chez mes parents. \\ Mon père est d'origine espagnol et s'est convertit à l'Islam et ma \\ maman c'est une française d'origine algérienne. \\ J'ai deux soeurs, une grande Imene de 27 ans, une petite Hyza de 21 \\ ans, et un petit frere Mehdi de 23 ans. \\ Ma famille c'est la prunelle de mes yeux $\bullet$ \\ En ce qui concerne les amis, comme beaucoup de personne, j'ai \\ énormément de connaissance mais une seule et vraie amie. \\ Imène ma chérie, ma moitié tout $\bullet$ On a grandit ensemble, donc elle \\ me connais par coeur tout comme moi je l'a connais par coeur. » $(2$, \\ partie 1)
}

Ces parties introductrices sont généralement «décrochées » de la suite par l'absence de numéro ou par une dénomination singulière comme dans la chronique (4) qui intitule la partie introductive "préface » et accompagne ce titre d'un commentaire d'auto-dérision « j'lol $\wedge^{\wedge}$. Elles commencent, dans six chroniques sur huit, par des salutations aux lectrices ( 2 sont en français, 2 sont arabe et 2 sont plurilingues), salutations que l'on ne retrouve plus au début des parties postées ensuite. Les deux chroniques qui ne s'ouvrent pas sur des salutations (3 et 6) partagent une autre caractéristique : le récit démarre dès la première partie tandis que dans les six autres le récit ne commence vraiment qu'avec la deuxième partie.

Dans quatre chroniques, la présentation est explicitement annoncée, soit par catégorisation de la séquence langagière : « (une petite) présentation » $(1,3$ et 7$)$, soit par la formule à valeur performative «j'me présente» (4). Dans deux autres chroniques (1 et 5), la présentation de soi est introduite directement, sans annonce préalable, par la formule «moi c'est », tandis que la dernière chronique (6) annonce explicitement que la séquence de présentation, du moins dans sa forme routinisée, sera esquivée : «je me présente pas », soulignant ainsi indirectement le caractère conventionnel et donc attendu des séquences de présentation.

Il y a ainsi sept séquences de présentation canoniques dans le corpus, dans lesquelles les narratrices / narrateurs donnent systématiquement leur nom et des informations sur leur âge au moment de l'écriture ou au moment du récit (en gras dans les extraits ci-dessous) :

« Moi c'est Soraya, une fille qui a atteint la 20aine et qui habite en région parisienne (...) ayant grandi depuis toujours dans une famille musulmane » (1)

«Une petite présentation (...) Je m'appelle Sabrina j'ai 24 ans (...) Mon père est d'origine espagnol et s'est convertit à l'Islam et ma maman c'est une française d'origine algérienne. (...) j'ai 24 ans » (2) 
« Déjà présentation : J'mappel Sabrina, Sasa , Sasou, Sabrinoush mdr j'suis Algeriene sahbi (...) j'ai 17 ans » (3)

« J'me présente moi c'est Camelia . J'suis d'origine Marocaine (...)

jvous rassure ma chronique n'est pas une histoire de gamine de 14 ans » (4)*

« Moi c Anissa Aujourd'hui j'ai 20 Ans mais je vais debuté a

lage de 14 ans.. (...) Je suis Iraqienne (...) Jabite en banlieu parisienne $»(5)$

« J'me présente pas A cette époque j'allais avoir 20ans j'sais plus

lol, j'étais un gars qui était posé avec une salope » (6)

«Petite présentation en deux-spi : /.../ Je m'appel Souad j'ai 23

ans, jeune fille musulmane d'origine tunisienne » (7)

«Je m'appelle Merveille (...) J'ai 20 ans, je suis zaïroise, trop fière de l'être » $(8)$

Dans six chroniques, la famille est présentée en détail, chaque membre de la fratrie étant nommé et caractérisé par un âge ${ }^{13}$. Concernant les amis, soit la présentation est faite dès la séquence initiale, soit elle est explicitement reportée à la suite du récit : «Mes shabs ? Vous ferez connaissance ac eux au fur et a mesure de l'histoire » (4).

Les origines migratoires de la famille sont annoncées de manière précise dans six chroniques (en italique dans les extraits de corpus ci-dessus) et suggérées dans la chronique (1), où la précision sur la confession musulmane de la famille et les prénoms les suggèrent. La chronique (6), la seule qui ne comporte pas de séquence de présentation à proprement parler, est plus atypique à bien des égards. C'est la seule du corpus qui appartienne au sousgenre «chronique écrite par un garçon». L'annonce explicite de «non-présentation» (parce que «les jaloux le feront pour moi ») n'empêche pas cependant le narrateur de donner son âge. Les informations que donnent souvent les chroniqueuses pour se décrire physiquement ou psychologiquement sont en revanche données à propos de l'héroïne, Fanny, dont le narrateur tombe amoureux.

La forme même des incipits contribue donc, dans le prolongement des titres, à catégoriser le récit qui démarre comme une chronique. Ces parties introductives jouent d'ailleurs de manière plus ou moins ouverte le rôle de "produit d'appel » pour fidéliser le lecteur. Le suspens sur le statut matrimonial de la narratrice au moment de l'écriture est introduit explicitement dans deux d'entre elles pour éveiller la curiosité du lecteur et le fidéliser :

« célibataire, en couple ou mariée? Telle est la question émoticône wink Vous le serez à la fin de ma chronique. » (1)

«Et oui oui oui, toute la ptite famille et mariée, et moi ? vous le saurez dans la suite... » (7)

Enfin, 5 parties introductives, sur les 8 du corpus, se terminent par une demande explicite pour que les lecteurs postent un «j'aime» qui rendra visible le nombre de fans et contribuera ainsi au succès de la chronique.

\subsection{Pacte autobiographique}

L'incipit est aussi, pour les chroniques analysées, le lieu d'un pacte autobiographique. Elles s'inscrivent en effet toutes dans la catégorie des chroniques «réelles », que les chroniqueuses et leurs lectrices opposent aux chroniques «fictives » où les autrices annoncent livrer un récit inventé, fruit de leur imagination ${ }^{14}$, La catégorisation comme chroniques «réelles » et «fictives » est souvent l'un des premiers éléments définitoires mentionnés pour présenter une chronique. Par exemple, lorsque l'animatrice du skyblog «Sam liseuse de chro» demande que les chroniqueuses lui envoient des éléments de 
présentation de leur chronique, elle retient les éléments suivants, confirmant au passage le rôle crucial des incipits dans la conquête des lecteurs :

\begin{abstract}
« Dans chaque article je vai présenter une chronique inshaallah, je dirais si elle est fini ou pas, si c'est une histoire vrai ou pas, si elle est sur Facebook ou Skyrock. Je metterais le Lien de la Chronique ainsi que la premiére partie si vous accrocher lisez la si non Mehlish (pas grave). »
\end{abstract}

Dans les chroniques « réelles » qui font l'objet de notre étude, les chroniqueuses s'engagent en revanche à raconter une partie de leur vie dans un «esprit de vérité » (Lejeune, 2005 : $31)$. Ce contrat de vérité donne le plus souvent lieu à un pacte autobiographique, placé dans l'incipit. Celui-ci est tout à fait explicite dans cinq des chroniques, où l'histoire est qualifiée de « vraie » $(2,5$ et 8$)$ ou «basée sur des faits réels » (4) par les chroniqueuses. Ce pacte de véracité est d'ailleurs noué directement avec le lecteur, comme le montrent les commentaires qui justifient les petits arrangements avec le réel et font valoir les risques qu'il y aurait à livrer une réalité brute :

«Je vais juste remixer les prénom pour gardé mon anonyma je veu pas mourire avant l'heure LOL.» (5)

« Certaines choses de l'histoire seront changés, gardons un peu de vie privé LOL. $\gg(6)$

D’autres raisons sont invoquées comme les limites de la mémoire, qui ne permet pas de se souvenir mot pour mot des dialogues (2 et 8 ). Dans tous les cas, le fait de reconnaître, au moment même de la formulation du pacte autobiographique, que la réalité ne pourra pas être livrée « brute » contribue à construire l'éthos d'un auteur honnête, parce que, comme le rappelle Maingueneau (cité par Amossy : 75) : «Ce que l'orateur prétend être, il le donne à entendre et à voir : il ne dit pas qu'il est simple et honnête, il le montre à travers sa manière de s'exprimer ». En reconnaissant que la vérité est parfois transgressée, les chroniqueuses placent le récit sous les auspices de la sincérité.

Néanmoins, un élément caractéristique du dispositif autobiographique reste absent des chroniques: le véritable nom propre du chroniqueur / de la chroniqueuse n'est jamais mentionné et il est au final impossible de confirmer l'identité entre celui ou celle dont est racontée la vie, celui ou celle qui dit « je » dans la chronique, et celui ou celle qui, derrière l'écran, écrit et publie la chronique. Les chroniqueurs / les chroniqueuses adoptent des stratégies d'anonymat et / ou de pseudonymat, qui renvoient à l'instabilité auctoriale qui caractérise les écritures sur internet, et l'on ne connaît pas leur identité IRL (In real life).

En ce sens, on peut rapprocher les chroniques d'autres formes d'écritures numériques, comme par exemple le courant des «mamans blogueuses », où des mères de famille font suivre « en direct » à leur communauté de lectrice le flux de leur existence (de l'annonce d'une grossesse à celle de la naissance du bébé par exemple) tout se dissimulant, pour la plus grande partie d'entre elles, derrière un pseudonyme. Mais si les chroniqueuses peuvent être inscrites dans une tradition qui est celle des écritures féminines de soi (il s'agit, pour les chroniqueuses, nous expliquent les participantes au forum de « ma-bimbo.com », de « se confier », "se vider l'esprit »), ce qu'elles ont à confier construit un univers référentiel caractéristique.

\title{
4.3 L'ancrage énonciatif des chroniques : entre histoire et discours
}

Récits autobiographiques à la première personne, les chroniques sont aussi fortement marquées par l'interactivité propre aux supports numériques. Comme dans d'autres formes d'écritures sur le web, s'établit en effet un contact direct entre la chroniqueuse et la 
communauté de lectrices qu'elle a fédérée. Celles-ci peuvent, par le jeu des «j'aime » et des espaces de commentaires proposés par l'interface de Facebook, non seulement réagir aux parties postées par la chroniqueuse, mais aussi échanger avec elle. Elles sont fréquemment sollicitées par la chroniqueuse elle-même, qui ne se cantonne pas à raconter la chronique, mais doit aussi animer la page Facebook sur laquelle celle-ci est placée ${ }^{15}$ : les chroniqueuses lancent des sujets de discussion, répondent aux questions de leurs lectrices, utilisent des appellatifs collectifs et affectifs pour s'adresser au lectorat et lui demander, par exemple, les « j'aime » qui feront la popularité de la page ${ }^{16}$.

Cette forme dialoguée des chroniques se remarque dès les parties d'ouverture, où les lecteurs et lectrices sont interpellés par des termes d'adresse (soulignés dans le corpus cidessous) ou des pronoms (gras dans le corpus ci-dessous), le jeu des salutations catégorisant ces narrataires comme des locuteurs prêts à se reconnaître dans une communauté de lecteurs multilingues et multiculturels :

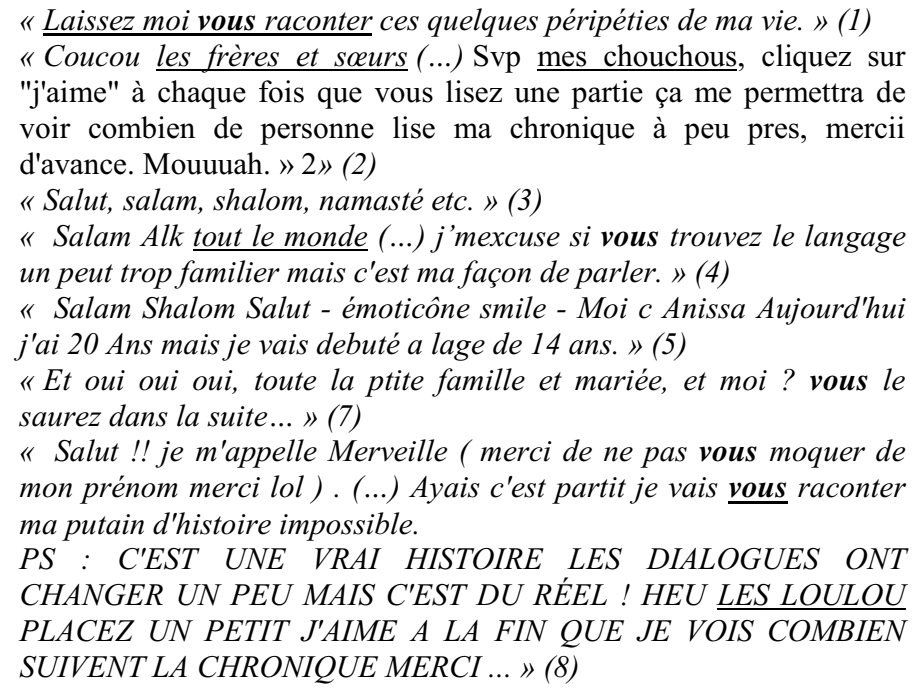

Dans le corps lui-même des chroniques, ce dialogue continue, et les adresses au lecteur sont fréquentes. Citons, à titre d'exemple, cet extrait de la chronique (6) où l'auteur/trice après avoir annoncé qu'il/elle va revenir sur des épisodes de la vie de sa mère qui vient de mourir, prévient le lecteur :

« J'envie de vous faire partager les bons moments que j'ai eu avec elle. Sa en fera peut-être chier certains. Mais maintenant vous m'connaissez. » (6, partie 19)

Cette caractéristique énonciative des chroniques les inscrit dans ce que Benveniste appelle le plan énonciatif du discours, celui qui s'ancre dans la relation «je-tu ». Il n'est donc pas étonnant que le passé composé et le présent soient les temps très largement privilégiés pour les séquences narratives.

Pour autant, l'examen des chroniques de notre corpus montre la présence du passé simple dans la moitié d'entre elles au moins. Dans la chronique (3), il est employé de manière quasi systématique lorsqu'il s'agit de faire « avancer le récit» :

"Je descendit de la voiture et monta directe chez moi par les escaliers
pour arrivé plus vite et m'enfermé dans ma chambre, la porte d'entré
était fermé bien sure je tapa comme une ouf sur la porte.» (3, partie 2)

Dans d'autres chroniques $(5,6$ et 8$)$, il alterne avec le passé composé et le présent ${ }^{17}$ : 


\author{
Alor je lui cria; \\ Ouaiii Garde la peche khouya (Frere) \\ Ce connard il ces meme pas retournéé rien puis il a tracé sa \\ route.. \\ J'avoue je vais pas mentire mais j'etais trop contente ... \\ Bref je re pars m'assoir sur notre banc de skoite. ») (5, partie
}

19)

On peut faire l'hypothèse que ces marques propres à ce que Benveniste appelle le plan énonciatif de l'histoire sont un moyen pour les chroniqueuses de s'inscrire dans une filiation plus littéraire, ou, comme le dit Maingueneau à propos des récits d'enfants qui combinent je + PS, une manière de «constituer son je en je textuel, personnage de narration » $(1981: 58)$.

Cet emploi du passé simple, selon des formes plus ou moins conformes aux normes prescriptives (orales et écrites ${ }^{18}$ ), est identifié comme un trait suffisamment saillant pour qu'il soit repris dans le titre d'un article du magasine en ligne Slate.fr (du 9 mai 2012), précisément intitulé : «Je me leva et je téléphonit:, les chroniques très «skyblog» de Facebook ».

\title{
4.4 Suspens, tuilage des épisodes et continuité narrative
}

Si l'on caractérise les chroniques du point de vue de ce que l'on appelle, à la suite de Bakhtine, la structure compositionnelle, on remarque qu'elles partagent toutes la caractéristique d'être très longues, et d'être découpées en de nombreuses «petites » parties (de quelques lignes à deux ou trois pages) qui s'enchaînent linéairement, postées au jour le jour, ce que permet et facilite le «post journalier» sur Facebook. Les chroniques constituent des productions volumineuses (123 parties pour Petite Cendrillon amoureuse du prince du ghetto, 100 parties pour Zaïroise love d'un rebeu qui couvrent dans les deux cas plusieurs centaines de pages), qui permettent de capter durablement l'attention de l'auditoire : leur écriture, tout comme leur lecture, apparaît comme une entreprise au long cours.

À ce titre, il semble pertinent de rapprocher les chroniques de l'écriture du feuilleton ${ }^{19}$ (Goetschel et alii, 2015). D'un point de vue émique, cette proximité entre feuilleton et chroniques ne semble pas explicitement formulée par les chroniqueuses ellesmêmes. Cependant, il est intéressant de remarquer que les séries télévisées sont, précisément, l'une des seules productions culturelles auxquelles font référence les chroniqueuses, tant dans le récit des chroniques elles-mêmes que dans le journal de leur page Facebook. Par exemple, lorsque la chroniqueuse de Petite Cendrillon amoureuse d'un prince $d u$ ghetto interroge ses lectrices à propos de l'intrigue de Vampire diaries :

\begin{abstract}
«Les filles, est-ce que l'une d'entre vous peut me dire pourquoi Elena (vampires diaries) est passée de Stefan à Damon ? Sah j'aime pas son bizness surtout qu'elle était amoureuse lol mais j'ai pas le temps de regarder les saisons avec les petits et quand j'ai vu ça il y a quelques temps je l'ai traité mdr mais je sais pas pourquoi elle est passée d'un frère à l'autre. Merci. Souad. » (7)
\end{abstract}

En outre, cette présentation des chroniques comme une déclinaison sur le web - ou plutôt une réactualisation - du feuilleton est présente dans plusieurs des articles consacrés aux chroniques que nous avons intégré à notre corpus :

« feuilletons des temps modernes, les chroniques Facebook fleurissent sur la toile. » (culturebox.francetvinfo.fr)

«Les filles des cités réinventent le feuilleton. »

(http://www.leparisien.fr) 
«Ces écrits qui réactualisent le principe du feuilleton sont un mélange de tranches de vie et de fiction publiés par des auteurs sous couvert d'anonymat. » (http://www.edilivre.com)

La longueur propre aux feuilletons est une caractéristique des chroniques partagée par d'autres écrits du web, notamment toutes les écritures de l'intime / de l'extime auxquelles s'intéresse Mackrous (2015). Les productions des «écriveuses du web » qu'elle étudie relèvent d'une sphère littéraire plus « légitimée » dans le monde académique, mais relèvent d'une même «écriture d'abondance »: il semble que l'absence de filtre éditorial pour atteindre son lectorat leur donne la même prolixité qu'aux chroniqueuses.

En revanche, cette écriture « abondante » des chroniques se distingue sur d'autres plans de l'écriture web. On n'y retrouve pas, notamment, les caractéristiques de la «mémoire métallique » (Orlandi cité par Dias 2015), mode organisationnel particulier de l'écriture de soi sur Facebook. En effet, selon Dias, l'écriture de soi sur Facebook se caractérise comme une mémoire fragmentaire impliquant «quantité » mais aussi «non-historicité ». Les événements n'y sont pas « historicisés »:

«Les marqueurs de temps auxquels nous sommes habitués, à savoir,
le présent, le passé, le futur, en rapport historique et en référence à un
événement faisant sens dans l'histoire (...) ne sont pas (...) des
éléments constitutifs. » (Dias $2015: 10)$

Cette « spatialisation de la mémoire » que décrit et analyse Dias ne caractérise pas du tout le mode d'écriture de ces « écriveuses » du web que sont les chroniqueuses.

Dans leurs récits, nous sommes dans un système classique de narration que Genette qualifierait d'ultérieure (1972). En cela les chroniques se distinguent d'autres formes de récits recensées par Genette et dont le Web a favorisé le développement, comme la narration simultanée (live-tweet par exemple). Les chroniques relatent des événements qui prennent toujours place dans une période révolue de la vie des chroniqueuses, période clairement située avant le moment de l'énonciation dans lequel s'ancrent les commentaires et autres formes d'échanges avec les lectrices. Les événements, les semaines, les saisons, les années de lycée se suivent et passent au fur et à mesure que sont postés les nouveaux épisodes.

L'écriture a posteriori des chroniques, très «classiquement » chronologique, tout comme l'inscription dans une écriture «feuilleton » contribue à expliquer (et/ou à rendre possible) le recours au suspens comme forme de «tuilage » entre les épisodes que l'on retrouve dans la plupart des chroniques et qui apparaît, de ce fait, comme constitutif du genre. Très souvent, à la fin des épisodes, un portable sonne, un SMS arrive, quelqu'un pose une main sur l'épaule de la chroniqueuse ... et il faut attendre l'épisode suivant pour que le suspens soit levé. Contrairement à la logique spatiale de continuité/contiguité entre les fragments de mémoire, qui prévaut dans la «mémoire métallique », toutes les chroniqueuses assurent une forme de tuilage entre les moments de mémoire qu'ils partagent avec le lecteur. Il peut s'agir, dans les jeux de suspens évoqués ci-dessus, d'achèvement énonciatif ou interactif : une partie s'achève au milieu d'une phrase, comme dans l'exemple ci-dessus, ou au milieu d'un échange et la partie suivante s'ouvre en terminant la phrase ou l'échange inachevé dans la partie précédente. Parfois les dernières lignes d'une partie sont reprises, à l'identique, ou avec reformulation, au début de la partie suivante. Dans la chronique (1), la seule du corpus qui n'interrompt pas les parties dans un moment de suspens, le lien entre les épisodes est néanmoins assuré. Différents procédés sont exploités pour inviter les lectrices à attendre (et à lire) l'épisode à venir. La partie 2 se termine sur : « Je remet mes écouteur l'air de rien ... Il faut que je vous parle de ce fameux Kery, mais promis au prochain épisode.... »; la partie 19 sur «A suivre inch'allah» et la partie 20 par « to be continued ». 


\subsection{Une écriture dialoguée}

Nous avons commenté ci-dessus l'ancrage énonciatif caractéristique des chroniques Facebook qui mettent discursivement en scène le dialogue entre narratrice/narrateurs et lectrices/lecteurs. Le récit lui-même laisse une très large place au dialogue entre protagonistes. Dans l'espace de cet article, nous pouvons seulement montrer l'ampleur du phénomène et indiquer les pistes d'analyse en cours.

Les propos écrits (via Facebook, les SMS ou parfois les lettres, lorsqu'un protagoniste se retrouve en prison) ou oraux (conversations en face à face ou par téléphone), insérés sous la forme d'un discours direct, prennent une place importante dans le texte. En effet, un décompte précis du texte correspondant à du discours direct, effectué pour les parties 19 de chacune des chroniques de notre corpus, aboutit à des pourcentages allant de $28 \%$ (Petite Cendrillon amoureuse du prince du ghetto) à $58 \%$ (La vida à la cité).

Dans la transposition des conversations entre les protagonistes des récits via MSN (pour les plus anciennes), SMS et Facebook, et de manière plus générale dans l'écriture du récit luimême, on retrouve des usages graphiques couramment attribués à des scripteurs « jeunes " et assez bien documentés aujourd'hui (voir par exemple Anis, 2006). Parmi les procédés décrits dans ces travaux, on peut citer l'agglutination morpho-syntaxique (5, partie 2 : «jvai tla ramné »), des réductions graphiques de digrammes et trigrammes (1, partie 20 : « est-ce kel est rentré de son rendez-vous ») ou encore l'utilisation de la valeur phonique épélative de certaines lettres comme le «K» par exemple (3, partie 2 « Kos »[kasos]), et les graphies consonantiques comme «mdr». Ces procédés d'écriture ne sont pas réservés aux parties dialoguées mais ils y sont généralement plus nombreux, bien qu'il y ait, concernant ces usages, des variations interindividuelles fortes qui émergent.

La part importante occupée par les dialogues, et notamment par les dialogues oraux synchrones (face à face ou téléphone), révèle une autre particularité des écrits des chroniques : la transposition, avec divers procédés scripturaux, de traits d'oralité. Ainsi, des lettres «e » non notées peuvent-elles transposer la non réalisation de schwas (《e muets »); la forme affirmative « ouais » connaît plusieurs variantes qui rendent compte de diverses prononciations attestées chez les jeunes comme «ué ». Divers procédés graphophonétiques comme un même graphème de voyelle répété pour noter l'allongement vocalique (1, partie 2 : «elle insiste. Moi : " Quoooooooooiiii ? J'écoute ma musique laa ! T'es relou " ») permettent également de rendre compte à l'écrit de la matérialité de l'oral.

De manière générale dans les chroniques, les dialogues sont marqués, d'un point de vue étique, par une hétérogénéité stylistique et linguistique. L'alternance codique y est ainsi omniprésente, commentée et/ou décodée au besoin pour le lecteur. Une étude plus systématique de ces alternances linguistiques et stylistiques est en cours (Bigot, Maillard et Lambert 2016) mais, à titre d'exemple, les extraits suivants illustrent la sensibilité de ces autrices à l'hétérogénéité langagière qui constitue une ressource stylistique :

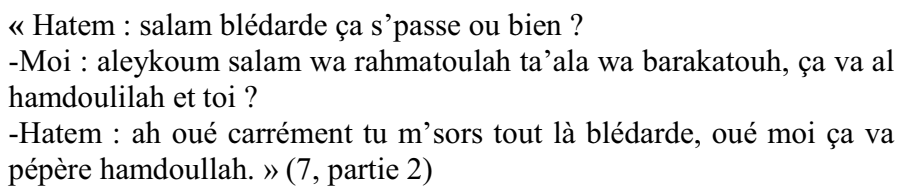

Cet échange révèle un des usages classiques de l'alternance codique (pour les formules rituelles de politesse, salutations, incantations etc.). La remarque adressée par Hatem à la narratrice souligne, avec une certaine ironie, la valeur identitaire que l'on peut attribuer à cette alternance ( le comportement est catégorisé comme typique d'un « blédard») expliquant implicitement au lecteur le sens global de la tirade et le dispensant ainsi d'une compréhension littérale. 
Dans la chronique 8, dont la narratrice est zaïroise, les emprunts à l'arabe sont fréquents et ne font pas l'objet de traduction. Ils semblent être considérés comme faisant partie du répertoire verbal partagé par l'autrice et ses lectrices. Dans la partie 3, lors d'une conversation avec son amie marocaine, la narratrice utilise par exemple " inch'allah », «mash'allah» et «faire le halal» (dans le sens de se marier) sans procéder à une quelconque «médiation» sémantique. En revanche lorsqu'elle utilise l'appellatif «Ma mundibu » pour s'adresser à son amie Hinda, elle en propose immédiatement une traduction entre parenthèse ${ }^{20}$ «(rebeu) ». Le lingala est présent à travers des mots d'emprunt, comme l'appellatif « leki » (petite sœur) ou des procédés d'alternance codique intraphrastique qui peuvent se révéler complexes ${ }^{21}$. C'est par exemple le cas dans cet extrait où la mère de la chroniqueuse s'adresse à sa fille : " en tous cas année wana merveille j'espère ko ko zua bac na yo hein et que mubulu eko sila sinan oko yeba nga très bien merveille ( $j$ 'espère que cette année tu auras ton bac et que c'est fini les bêtises sinon tu vas bien me connaître) » (8, partie 5). Les chroniqueuses puisent aussi largement dans un matériau langagier marqué par l'oralité populaire et l'hétérogénéité linguistique de leurs réseaux de sociabilité/socialisation. Ainsi, dans cet extrait où des amis parlent de mariage, entend-on, par un jeu de stylisation, la variété de français de celui qu'ils catégorisent comme «blédard», et dont le trait caractéristique retenu ici est le suremploi du son vocalique [i] :

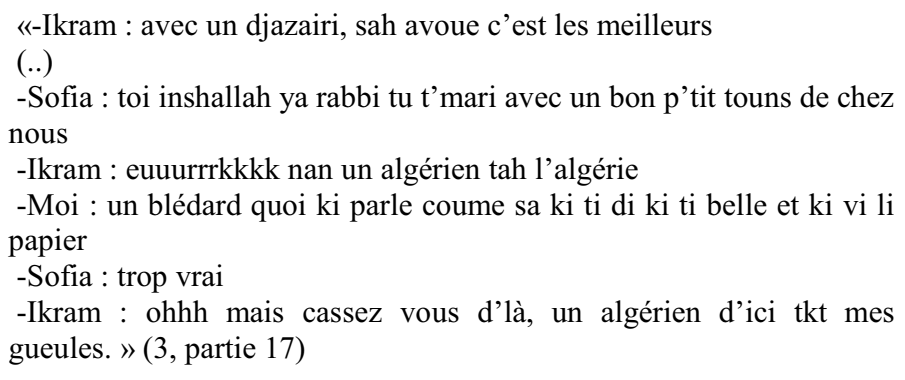

\subsection{Les thèmes caractéristiques du genre}

Toujours dans une démarche de croisement des perspectives étiques et émiques, nous nous appuyons, pour cette étude des thèmes caractéristiques du genre, sur l'étude des répertoires de chroniques et sur les chroniques elles-mêmes, plus particulièrement sur les titres et les incipits qui sont des lieux révélateurs des thèmes privilégiés.

Après avoir recensé les thèmes dominants, mobilisés dans les répertoires de chroniques pour les « ranger» dans des albums séparés, nous évoquerons l'importance des réseaux de socialisation familiaux, amicaux et amoureux, mis en discours par les chroniqueuses pour construire des récits d'apprentissage. Dans un troisième temps, nous montrons comment la cité, et la mixité interculturelle qui la caractérise, constituent un des thèmes centraux et caractéristiques de l'ensemble des chroniques

\subsubsection{Les thématiques identifiées par les chroniqueuses et leurs lectrices}

Le contenu sémantique des chroniques est assez précisément balisé. Comme l'indique Axellya, l'une des participantes du site «ma-bimbo.com », une chronique :

«c'est juste une histoire que des jeunes filles (ou jeunes hommes) racontent, en general leur vecu. /.../ /../ En general, les chroniques parles de longues histoire d'amours complexe, de drame ...Bref, un vrai film a l'eau de rose sur ecran. Sauf qu'il y d'autres sujets, il y a des 
sujets qui parle de la mort, de la religion, des histoires fausses egalement. »

Une analyse du mode de classement adopté par les administratrices des «répertoires de chroniques » permet de mettre en évidence de manière plus précise les thématiques privilégiées par les chroniques, ainsi que l'importance de ce critère lorsqu'il s'agit de regrouper entre elles des chroniques comme elles le font. Ainsi, sur 14 pages Facebook de ce type, nous avons pu recenser 12 albums qui réunissent des chroniques portant sur des thématiques amoureuses, et donnent accès à 484 chroniques $^{2}$, confirmant donc l'orientation thématique mise en évidence par Axellya («de longues histoires d'amour complexe »). sept chroniques de notre corpus relèvent de ce sous-genre. Autre thématique, qui recoupe parfois la précédente, celle de la violence faite aux femmes : on comptabilise au total huit albums qui réunissent 92 chroniques sous les intitulés "mariage forcé », «femmes battues", "viol, kidnapping ». L'univers des gangs et de la mafia est très présent : dans les répertoires examinés, 6 albums recensent au total 622 chroniques autour de thèmes tels que «thug life », «thug love », "Chroniques de gang, kidnapping mafia prison ». Enfin, la religion est elle aussi présente, quoique dans une moindre mesure : elle apparaît dans trois albums («chroniques qui parlent du dine», «spécial chroniques religion » et « chroniques sur la religion ») regroupant 28 chroniques. La chronique (1) de notre corpus relève de ce sous-genre.

\subsubsection{Mes amis, ma famille, mes amours : des récits d'apprentissage}

L'étude des incipits a permis de mettre en valeur le rôle de la famille, premier cercle de socialisation, au cœur de toutes les chroniques étudiées, y compris des deux qui ne procèdent pas à une présentation détaillée des membres de la famille en début de récit. Les amis constituent le deuxième cercle de socialisation à la description duquel les chroniqueuses s'attachent. Ce cercle des amis, dont une partie au moins constitue des « amis d'enfance », constitue un espace d'émancipation de la famille présent également dans tous les récits. Quand ce ne sont pas la famille (que l'on présente en premier) et les amis (avec lesquels on passe le plus de temps) qui occupent le devant de la scène des récits, c'est la / les relations amoureuses qui sont thématisées comme voie d'entrée dans le monde adulte. quatre des huit chroniques thématisent l'amour dans le titre (2, 6, 7 et 8$)$. Le rôle des relations amoureuses dans cette émancipation du premier cercle familial, (éventuellement mis en regard de l'entrée dans un autre cercle familial, celui de la belle-famille) constitue donc une thématique très présente dans ces récits. L'école, ou plus exactement le lycée, constitue également un espace de socialisation, transitoire entre la famille et l'entrée dans le monde adulte et très présent dans les récits. Dans notre corpus, le lycée est mentionné au moins une fois, dès les deux premiers chapitres, dans sept des huit chroniques. La seule dont il soit absent est la (2) qui raconte l'histoire d'une jeune femme déjà entrée dans le monde du travail.

\subsubsection{Des femmes dans la cité et dans la mixité}

Nous avons déjà souligné que les chroniques sont écrites - et lues - très majoritairement par des filles. Les chroniques écrites par des garçons et/ou dont le personnage principal est un garçon sont rares. Des albums spécifiques leurs sont d'ailleurs consacrés dans les répertoires de chroniques examinés : «chroniques de gars» (trois albums et 144 chroniques), « chroniques de meks » (deux albums et 20 chroniques). Le lectorat « visible » 
est également essentiellement féminin. Cela frappe immédiatement si l'on regarde les profils des lecteurs/trices et qui sont les signataires des commentaires. Parfois, comme dans la (7), l'adresse aux lecteurs dans le cours du récit est en fait une interpellation " genrée »: « les filles vous voyez quand vous casser avec votre ex peut importe la raison même si la rupture (...) ». L'univers virtuel des chroniques est donc largement celui d'un « entre soi » féminin qui se déploie sur la toile.

De manière générale, les narrateurs/trices se présentent comme femmes, jeunes, vivant dans un environnement urbain populaire, dans des familles dont nous avons déjà souligné qu'elles partagent toutes un passé migratoire. La rencontre interculturelle, les amitiés entre jeunes issus de diverses origines, et les amours « interethniques » apparaissent comme une thématique centrale de nombreuses chroniques. Deux titres: Chronique de Sabrina love d'un rebeu (2) et Zairoise love d'un rebeu (8) sur les huit du corpus thématisent la question du couple interethnique. Dans la chronique (3), la question intervient dès le chapitre 2 où la sœur de la narratrice Sabrina, rencontre en cachette son amoureux sénégalais. La chronique (6) traite également de cette question: le narrateur marocain y est amoureux d'une ivoirienne. Les meilleur(e)s ami(e)s des chroniqueuses, les garçons dont elles tombent amoureuses construisent l'image d'une cité où les relations "interethniques » sont nombreuses, peuvent faire l'objet de préjugés (chez la génération des parents notamment) mais aussi de discussions, de fantasmes et sont l'occasion de nombreuses expériences interculturelles et plurilingues pour les protagonistes. Dans la chronique (7), la question des bonheurs et difficultés du couple mixte est longuement discutée avec les sœurs et amies de la narratrice, et l'acceptation de l'amoureux maghrébin par le grand frère et la mère de la narratrice occupe plusieurs épisodes. Dans les répertoires de chroniques, des intitulés d'albums tels que « chronique amour tissmey, babtou, renoi, rebeu » ou « chro love renoi » témoignent de l'importance de la rencontre interculturelle. Dans certaines chroniques (chronique 2 de notre corpus par exemple), c'est la chroniqueuse qui met en avant son propre métissage. Cette caractéristique est d'ailleurs suffisamment fréquente pour qu'une parodie de chronique ${ }^{22}$ la tourne en dérision :

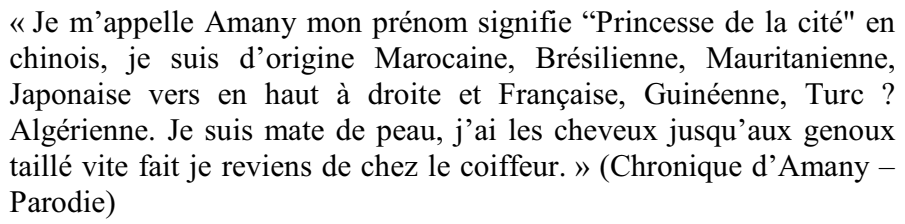

La diversité des origines migratoires n'est pas seulement thématisée à travers les catégorisations «ethniques » des personnages, elle est aussi rendue manifeste dans les pratiqiques plurilingues déjà évoquées, et notamment dans les salutations initiales en début d'épisode 1, qui peuvent mêler jusqu'à 4 langues : français, arabe, hébreu, pour la (5) et la (7), auquel s'ajoute le sanskrit pour la (7). On retrouve ici une de ces pratiques « intragroupales » des jeunes de milieu urbain, que décrivait J. Billiez, dans un article de 1992 où elle montrait la fonction emblématique de cette hétérogénéité montrée à travers le recours à des répertoires plurilingues.

L'étude des séquences de présentation, et des titres, révèle enfin que les chroniqueuses considèrent leur environnement urbain comme un élément central du récit. Les titres des chroniques : Cerise sur le ghetto (3), La vida a la cité (4), Cendrillon amoureuse du prince $d u$ ghetto (7), mais aussi la présentation des lieux de résidence (en région parisienne, en banlieue parisienne, dans un lycée ghetto) et surtout les récits eux-mêmes font des cités (que l'on quitte, où on s'installe, dont on caractérise les mœurs, qui permettent de catégoriser les personnages etc.) des personnages à part entière des récits. 


\section{Conclusion}

Cette étude exploratoire a permis de montrer que les chroniques Facebook, malgré leur caractère très récent, constituent un genre de discours avec ses conventions propres. Elle a permis également de commencer à comprendre comment ce genre a pu émerger. L'intensité des interactions entre chroniqueuses et lectrices de la communauté discursive qui s'est constituée autour des chroniques Facebook nous semble avoir contribué à la stabilisation rapide du genre. Au-delà de cette stabilisation, notre étude révèle que son émergence s'acccompagne de l'émergence de sous-genres, plus ou moins clairement identifiés par la communauté discursive, et de productions catégorisées comme relevant du genre « chronique » mais partiellement transgressives des conventions établies. Ces phénomènes complexifient l'étude du corpus mais sont pleinement constitutifs du phénomène d'émergence d'un genre, de la manière dont les membres de la communauté discursive investissent, de manière personnelle et potentiellement créative, l'espace discursif qu'ils se créent.

La poursuite de l'étude des chroniques et des discours qui les commentent permettra de s'interroger sur les normes langagières que convoque cette communauté discursive : quelles sont ces normes? Pourquoi sont-elles convoquées ?

Comme le rappelle Sophie Moirand, s'appuyant sur Bakhtine, c'est la diversité des sphères d'activités humaines qui explique la diversité des genres discursifs. Le renouvellement de ces sphères d'activité, et des supports/medias de communication implique donc un renouvellement des répertoires génériques (Moirand 2009 : 92). Cette première étude exploratoire des pratiques discursives de la communauté d'internautes qui se forme autour de l'écriture et de la lecture des «chroniques"semble du point de vue de l'étude des modalités de construction d'un genre nouveau, particulièrement riche et prometteuse.

D'une part il s'agit d'une communauté jeune, que l'on qualifie souvent de « digital natives » et dont les expériences discursives sont très fortement concentrées sur internet. L'étude de la «traçabilité des domaines de mémoire » (Moirand 2009 : 92) qui constituent le tissu interdiscursif des chroniques s'en trouve donc fortement enrichie et facilitée par la disponibilité en ligne de l'essentiel de ces traces repérables grâce aux moteurs de recherche. Mais cette richesse en complique l'étude. La complexité des réseaux discursifs dans lesquels le corpus étudié se trouve pris multiplie à l'infini les traces des domaines de mémoires pertinents pour comprendre comment se constituent les réseaux de signification dans lesquels leur sens peut être co-construit.

Cette première étude nous a permis d'aborder les productions des chroniqueuses et de leurs lectrices, non pas sous l'angle des écarts à la norme linguistique (morphosyntaxique notamment), de la transgression des règles orthographiques, ou même de l'entrée peu défendable d'un point de vue théorique du «langage des jeunes » mais de la capacité des chroniqueuses à investir un genre discursif nouveau et à répondre à ses attentes et peut-être pour certaines, à jouer avec les «règles du genre». La suite des recherches sur les chroniques devrait confirmer l'intérêt d'une approche par le prisme du genre pour étudier la compétence de communication telle que définie par Hymes (1984).

\footnotetext{
${ }^{1}$ Dans la suite de l'article, nous avons choisi d'évoquer les membres de cette communauté au féminin (chroniqueuses, lectrices etc.), les hommes en étant quasiment absents. Par ailleurs, on soulignera que cette communauté est en fait constituée d'un réseau de communautés.

${ }^{2}$ Nous remercions Judith R., lectrice de chroniques dans ses années de collège-lycée. Elle a très vite compris l'intérêt que pouvait revêtir cette pratique discursive pour des études linguistiques et nous a convaincues d'en faire un objet de recherche. Elle a accompagné nos premiers pas dans cet univers virtuel.
} 
${ }^{3}$ Le numéro indiqué devant chaque titre de chronique est utilisé dans cet article pour donner les références des extraits analysés. L'orthographe des titres et des extraits des chroniques est conforme à celle utilisée par les autrices.

${ }^{4}$ Concernant ces deux chroniques, voici les informations qui rendent compte de leur visibilité sur le net en juin 2015 :

7 : Page commencée le 11-10-2011, toujours en activité, Facebook : 42343 j'aime.

8 : Page commencée le 28-01-2011-dernier message 25 nov 2012, Facebook : 20452 j'aime.

${ }^{5}$ La comptabilisation précise est difficile, car certains « albums » sont des publicités, des chroniques isolées, des concours montages. On peut faire la même remarque pour le nombre de chroniques recensées. 2695 chroniques sont citées dans ces 80 albums, mais d'un répertoire à l'autre (et parfois même à l'intérieur d'un même répertoire, d'un album à l'autre), les chroniques peuvent être recensées plusieurs fois.

6 - Astrid (2014). http://www.edilivre.com/communaute/tag/laila-1/\#.Vm19zHvgNJk (publié le 19/06.2014)

- Clairouin, O. (2012). Je me leva et je téléphonit», les chroniques très "skyblog" de Facebook. http://www.slate.fr/story/54701/chroniques-facebook-skyblogs (Mis à jour le 09.05.2012, publié le 09/05/2012).

- De Sepausy, V. (2014). Les chroniques Facebook commencent à intéresser les éditeurs. https://www.actualitte.com/article/lecture-numerique/les-chroniques-facebook-commencent-ainteresser-les-editeurs/46579 (publié le 27/01/2014).

- Houot, L. (2014). Quand les éditeurs s'emparent des chroniques Facebook pour en faire des livres. http://culturebox.francetvinfo.fr/livres/romans/quand-les-editeurs-semparent-des-chroniquesfacebook-pour-en-faire-des-livres-148541 (Mis à jour le 09/02/2014, publié le 24/01/2014)

- Les filles des cités réinventent le feuilleton. http://www.leparisien.fr/espace-premium/air-dutemps/les-filles-des-cites-reinventent-le-feuilleton-11-01-2014-3482563.php (publié le 11/01/2014).

${ }^{7}$ C'est dans une recherche portant sur des rapports de signalement d'enfants en danger que Cislaru et Sitri ont introduit cette notion. La valeur « cadrante » du corpus complémentaire qu'elles ont recueilli prend un sens plus fort que dans notre cas, car il s'agit de textes juridiques ou de "guides du signalement» produits par les institutions, et qui cadrent, au sens propre, les pratiques des professionnels. La valeur «cadrante » des discours méta que nous intégrons est moins explicite et c'est donc entre les chroniques elle-mêmes qu'il faudra étudier (aussi) les dynamiques interdiscursives.

${ }^{8}$ Celle-ci n'est pas avérée dans les chroniques de notre corpus mais se retrouve dans d'autres chroniques comme : https://www.wattpad.com/168791805-chronique-leyla-\%C3\%A0-la-vie$\% \mathrm{C} 3 \% \mathrm{~A} 0$-la-mort-saison-2-partie/page/2

https://www.facebook.com/270444106413835/photos/a.270581156400130.1073741830.2704441064 $13835 / 271179466340299$.

${ }^{9} \mathrm{D}$ 'autres commentaires - ainsi que nos propres investigations - corroborent les propos d'Axellyah et semblent indiquer que le genre se constitue au tournant des années 2009-2010. Une recherche via google trend de la fréquence des recherches « chronique Facebook » montre que celles-ci apparaissent à partir d'août 2009, croissent ensuite régulièrement, puis diminuent à partir de juillet 2014.

${ }^{10}$ Notamment, en 2006, on note la création d'un blog par une journaliste et un photographe, Aline Leclerc et Elodie Ratsimbazafy, intitulé "Chronique d'une banlieue ordinaire » (http://lacourneuve.blog.lemonde.fr/2010/06/11/chronique-dune-banlieue-ordinaire/), introduit en ces termes par les blogueuses :

«Ce blog démarre aujourd'hui, mais voilà plusieurs mois que notre reportage a commencé à la cité des 4000 de La Courneuve, dans le département de la Seine-Saint-Denis. L'idée vient d'une question simple de journaliste : comment parler des quartiers sensibles autrement que ponctuellement, lors d'un fait divers, de la publication d'un rapport, de la visite d'un ministre qui se rappelle leur existence. 
Quelle est la vie de ces quartiers le reste du temps ? /.../ Nous avons voulu voir ce précipice de plus près. Depuis deux mois, à La Courneuve, nous croisons chaque jour des gens ordinaires (...) Le quotidien de toutes les villes de France. Ou presque $»$.

La même année, un article du Parisien du 25 octobre 2006 (http://www.20minutes.fr/societe/11663820061024-selection-blogs-banlieue) présente plusieurs blogs évoquant la banlieue dans son quotidien. Parmi les blogs cités, « Made in Aulnay », tenu par Zineddine Chenoufi et Ouardi Taguia, et hébergé par liberation.fr. Ces deux habitants d'Aulnay-sous-bois ont tenu « des chroniques de la vie quotidienne dans l'une des villes les plus touchées par les émeutes de l'année /précédente/ ». Dans le premier post, ils disent avoir voulu « décrire ce /qu'ils voyaient/ de leur fenêtre ».

${ }^{11}$ Collectif "Qui fait la France», Chronique d'une société annoncée, Stock, 2007. Le collectif rassemble des auteurs comme Faïza Guène, Karim Amellal, Jean-Eric Boulin, Khalid El Bahji, Habiba Mahany, Mohamed Razane, Thomté Ryam, Samir Abdel ou Mabrouck Rachedi. La citation est extraite du site de l'éditeur (http://www.editions-stock.fr/chroniques-dune-societe-annoncee9782234060739)

${ }^{12}$ Soulignons toutefois que la position sociale de la lectrice de chroniques qui nous a servi de "go between" n'est pas celle des autrices de chroniques, du moins telle que ces dernières mettent en scène leur socialisation adolescente dans des milieux urbains socialement périphérisés. Cette lectrice nous a d'ailleurs expliqué qu'il était inimaginable qu'elle se présente sur la toile comme "lectrice de chronique". Il est donc difficile d'apprécier la part que peut jouer ce type de lectorat, qui lit les chroniques avec la curiosité qui anime la lecture des récits de voyage exotique, dans l'imaginaire des autrices et dans la réalité des pratiques de lecture.

${ }^{13}$ Pour des raisons de place, ces présentations ne sont pas reproduites.

143 albums recensent ainsi des chroniques « réelles » (125 chroniques au total) et 4 des chroniques « fictives » (109 chroniques au total).

${ }^{15}$ Ce rôle pouvant être, pour les chroniques les plus populaires, délégué à des «administratrices », lectrices et / ou amies de la chroniqueuse.

${ }^{16}$ Néanmoins, il ne s'agit pas à proprement parler d'une écriture collaborative, comme il peut en exister sur le web. Tout au plus trouve-t-on parfois des demandes d'éclaircissement sur une scène ou le devenir d'un personnage abandonné.

${ }^{17}$ On ne peut pas exclure que sur les milliers de pages que représentent les chroniques où le passé simple est utilisé, on puisse montrer des régularités fonctionnelles dans la distribution du passé composé et du passé simple.

${ }^{18}$ Les chroniqueuses forment les passés simples de la première personne en calquant la forme phonique (pour les verbes du premier groupe) ou orthograhique (pour les autres verbes) de la troisième personne : « je monta », « je cria », « je tapa », « je descendit ». Sans doute que ces formes leur sont-elles mieux connues.

${ }^{19}$ Une autre caractéristique du feuilleton populaire que l'on retrouve dans les chroniques est la forte stéréotypie des personnages (le voyou séducteur, le grand frère violent, la copine extravertie ...), des situations (décès d'un proche - parent ou ami - par exemple) ou des modalités du récit (voir ci-après pour le caractère très ritualisé des incipits). Enfin, comme les séries télévisées, une des seules références de "production fictionnelle " fréquemment commentée par les chroniqueuses, les chroniques peuvent aussi se décliner en saisons successives.

${ }^{20}$ Tous les recours au lingala font l'objet d'une traduction.

${ }^{21}$ Dans cet énoncé, les langues imposent tour à tour leurs contraintes syntaxiques comme le montre la postposition du déterminant «wana », conforme à la syntaxe du lingala et la structuration globale de l'énoncé en français « en tout cas....j'espère.....et que....».

${ }^{21}$ La chronique est annoncée comme parodique par le commentaire suivant : « Cette chronique à était créé pour but de montrer que quelque chronique sont parfois ou bien même toujours dans "l'excès ". Tous sa dans la rigolade bien sûr ! A ne pas prendre au sérieux. » (https://www.facebook.com/pages/Chronique-dAmany-Parodie-/1569783319910684) 


\section{Références bibliographiques}

Anis, J. (2006). Communication électronique scripturale et formes langagières. Réseaux humains / réseaux technologiques, 4 http://rhrt.edel.univ-poitiers.fr/document.php?id=547.

Beacco, J.-C. (2004). Trois perspectives linguistiques sur la notion de genre discursif. Langages, 153, 109-119.

Benveniste, É (1966). Problèmes de linguistique générale, Paris : Gallimard.

Bigot, V., Maillard, N. et Lambert, P. (2016). «Quand les ados font parler les adultes. Processus de différenciation des pratiques langagières des jeunes et des adultes dans les chroniques facebook », communication au colloque Vals-Asla, Genève, janvier 2016.

Billiez, J (1992). Le parler véhiculaire interethnique de groupes d'adolescents en milieu urbain. In Chaudenson, R. éd. Des villes et des langues, actes du colloque de Dakar, Paris, Didier.

Billiez, J. \& Lambert, P. (2008). Dans les coulisses de la (socio)linguistique urbaine française : le silence criant des filles, in Moussirou-Mouyama A. (éd.) Les boîtes noires de Louis-Jean Calvet, Paris : Editions Ecriture, 364-370.

Charaudeau, P. et Maingueneau, D. (2002). Dictionnaire d'analyse du discours. Paris : Le Seuil.

Dias, C. (2014). L'écriture du fragmentaire quotidien entre mémoire discursive et mémoire métallique. Itinéraires (en ligne), 2014-1. mis en ligne le 05 février 2015, consulté le 3 septembre 2015. URL : http:// itineraires.revues.org/2289 ; DOI : 10.4000/itineraires.2289.

Genette, G. (1972). Figures III. Paris : Seuil.

Goetschel, P. \& alii (2015). Écritures du feuilleton. Sociétés \& Représentations, 39.

Hymes, D.H. (1984). Vers la compétence de communication. Paris : Hatier-Crédif.

Jauss, H.-R. (2001). Pour une esthétique de la réception. Paris : Gallimard.

Kerbrat-Orecchioni, C. (1990, 1992 \& 1994). Les interactions verbales. Tome 1, 2 \&3. Paris : Armand Colin.

Lejeune, P. (2005). Signes de vie. Le pacte autobiographique 2. Paris : Seuil.

Lucken, C. (2002). Chronique. In Aron P. et alii éd., Le Dictionnaire du littéraire. Paris : PUF, 90-91.

Mackrous, P. (2014). Écriveuses du web : abondance et remix. Itinéraires (en ligne), 1). mis en ligne le 04 février 2015, http://itineraires.revues.org/2276

Maillard, N. et Bigot, V. (2015) : «Les chroniqueuses facebook », communication à la journée d'études Voix de femmes, projet Gedi, MSH Germaine Tillon, Angers, 16 juin 2015.

Maingueneau, D. (1981). Approche de l'énonciation en linguistique française, Paris : Hachette.

Maingueneau, D. (1996) : Les termes clés de l'analyse du discours. Paris, Seuil.

Maingueneau, D. (2007). Genres de discours et modes de généricité. Le français aujourd'hui 159, 29 35 (www.cairn.info/revue-le-francais-aujourd-hui-2007-4-page-29.htm.).

Moirand, S. (2003). Quelles catégories descriptives pour la mise au jour des genres du discours ? In Kerbrat-Orecchioni C. Journée scientifique sur Les genres de l'oral, dans le cadre de la formation doctorale de sciences du langage (conférence éditées en ligne sur le site du GRIC (UMR ICAR), université Lyon 2-Lumière et ENS LSH [http://gric.univlyon2. fr/Equipe1/actes/journees_genre.htm].

Moirand S. (2007). «Le modèle du cercle de bakhtine à l'épreuve des genres de la presse ». Linx(56). pp. 91-108. 
Mourlhon-Dallies, F. (2007). Communication électronique et genres du discours. Glottopol, 10, 2007, 11-23. http://glottopol.univ-rouen.fr/telecharger/numero 10/gpl10 01mourlhon.pdf

Münchow von, P. (2007). Le genre en linguistique de discours comparative. Stabilités et instabilités séquentielles et énonciatives. LINX, 56, 109-125.

Münchow von, P. (2010). Langue, discours, culture: quelle articulation? (2 ${ }^{\text {ème }}$ partie). Signes, Discours et Sociétés [en ligne], 4. Visions du monde et spécificité des discours, janvier http://www.revue-signes.info/document.php?id=1452. ISSN 1308-8378.

Olivier De Sardan, J.-P. (1998). Émique. L'Homme, 38, 151-166.

Petit, G. \& Beacco, J.-C. (2004). Le lexique ordinaire des noms du dire et les genres discursifs. Langages, 38(154), 87-100.

Schaeffer, J.-M. (1989). Qu'est-ce qu'un genre littéraire? Paris : Seuil.

\section{- 2 à 4 mots-clés}

littéracies numériques - genres discursifs -émique/étique - 